Retraction

\title{
Retracted: A Rare Case of Chronic Appendicitis Superimposed on an Incarcerated de Garengeot Hernia Prospectively Identified on Computed Tomography
}

\author{
Case Reports in Surgery \\ Received 26 July 2018; Accepted 26 July 2018; Published 23 October 2018 \\ Copyright (C) 2018 Case Reports in Surgery. This is an open access article distributed under the Creative Commons Attribution \\ License, which permits unrestricted use, distribution, and reproduction in any medium, provided the original work is \\ properly cited.
}

At the request of the authors, the article titled "A Rare Case of Chronic Appendicitis Superimposed on an Incarcerated de Garengeot Hernia Prospectively Identified on Computed Tomography" [1] has been retracted. There was disagreement between the authors in regards to the pathology results and final diagnosis, as chronic appendicitis could not be determined on the pathology. There is a De Garengeot hernia, however, chronic appendicitis is not a pathologic diagnosis and therefore there are no pathologic criteria to diagnose such an entity.

\section{References}

[1] A. Goldbach, P. Hota, A. Czulewicz, C. Burgert-Lon, and O. Agosto, "A rare case of chronic appendicitis superimposed on an incarcerated de Garengeot hernia prospectively identified on computed tomography," Case Reports in Surgery, vol. 2018, Article ID 5324320, 6 pages, 2018. 